\title{
Neuropsychobiology 1988;19:218-219
}

\section{Subject Index}

Abortive sequences 212 Acetylcholine 139 Acetylcholinesterase 149 Affective disorder 9,35,61 Alcoholism 79 Allopurinol 51 Alzheimer's disease 64,69 Amitriptyline 79 Amphetamine 97 Anticonvulsant drugs 104 Antidepressants 25, 116, 125, 146 Antidëpressive drugs 20

$$
\text { - effect } 20
$$

Anxiety $17,79,86,195$

Attention 90

Automatic analysis 212

Aversion 86

Barbiturate 86 Basal ganglia 6 Behavior 202 Benzodiazepine(s) 108, 186

- receptor ligand 195

Bioamines 149

Bioavailability 186

Bipolar II 61

Blood-brain barrier 180

Blood levels 202

Brainstem auditory evoked potential 104

Calcium 149 Carbamazepine 104 Catecholamines 192 Cats 212

Central synapses 139 Cerebrospinal fluid 64, 121 Chemical kindling 195 Chlorimipramine 20 Cholinergic-adrenergic balance 35 Chorea 6 Chronic pain 25 Chronobiology 176 Circadian rhythm 12 Clomipramine 25 Cognitive therapy 45 Convulsions 195 Corticosteroids 17 Corticotropin-releasing hormone 40

Dementia 135

Depression $1,17,40,45,61,73,79,125$ 
Depressive symptomatology 25

Dexamethasone suppression test 73

Diabetes 146

Diazepam 90

Diphenhydramine 90

Diurnal and seasonal variation 9

Dm 158

Dopamine 17,180

- receptor 180

Drug effects 158

Eating disorders 64, 121

EEG 108

-, psychotropic drugs 169

- reactivity 169

Electroencephalogram 54

Electroencephalography 116

Encoding 158

Epilepsy 5

Erythrocyte membrane transport 125

Erythrocytes 12

Event-related potentials 158

Factor analysis 135 FG7142 195 Flurazepam 108

GAB A B receptors 146 Galanin 64

Gamma-aminobutyric acid 139 Glutamate 139 Glycosaminoglycan polysulfate 135

Haloperidol 97 Healthy volunteers 202 Hemisphere, left and right 180 Hjorth's descriptors 212 Homovanillic acid 17 Hypomania 61

Idiopathic pain syndromes $25\left[{ }^{3} \mathrm{H}\right]$-Imipramine 69 Italian patients 1

Laterality 45 Locomotor behavior 97

Major depression 169 Maprotiline 20, 25 Membrane transport 12 
Memory 158 Menstruation 12 Mesolimbic dopamine 97 Metabolites 202 Methylphenidate 90

Minnesota Multiphasic Personality Inventory 149 Monoamine oxidase B activity 149 Mood 90 Mouse 180

Neural networks 108 Neurotransmitters 139 Nicotine 54 Nootropics 139, 158 Normal sequences 212

Pain 202

Pattern recognition 108

Peptides 121

Performance 90

Personality 149

Pharmacodynamics 202

Pharmacokinetics 186

Pharmacotherapy and cognitive therapy 45

Phenobarbital 104

Physostigmine syndrome 35

Piracetam 139

Plasma biopterin level 61

Platelet $3 / 4$ imipramine binding 9

Platelets 69

Prazepam 186

Pregnancy 12

Proteins 149

Psychometric methods 40

Psychopathology 6

Psychosis 176

Quantitative EEG 169, 202 Quazepam 108

Rat 86, 116

Ratings of perceived exertion 192

Remission phase 61

Respiration 202

Retrieval 158

Schizophrenia 45, 176 Secobarbital 90 Sensation seeking 149

Subject Index 
Serotonin 69

Severe epilepsy 51

Single doses 202

Sleep 108

- deprivation 73

- deprivation 73

Smoking 54

Somatic complaints 79

Spectral analysis 108

Subcutaneous morphine 202 
Sublingual prazepam 186 Susceptibility to punishment 149 Symptomatic phase 61 Symptoms 1 Synaptosomal uptake, dopamine 180 Systemic lupus erythematosus 17

Telemetry 116 Tianeptine 79 Tolerance 97 
Triazolam 108

L-Tryptophan 12, 125

Type A/B psychobiological reactivity 192

L-Tyrosine 12, 125

Na-Valproate 104

Water intoxication 176 Weight gain 176 Withdrawal 86 